\title{
Molecular Signature and Immune Landscape of HCV-Associated Hepatocellular Carcinoma (HCC): Differences and Similarities with HBV-HCC
}

\author{
Davide De Battista' \\ Fausto Zamboni ${ }^{2}$ \\ Hannah Gerstein \\ Shinya Sato' \\ Tovah E Markowitz ${ }^{3,4}$ \\ Justin Lack ${ }^{3,4}$ \\ Ronald E Engle (D) \\ Patrizia Farci' \\ 'Hepatic Pathogenesis Section, \\ Laboratory of Infectious Diseases, \\ National Institute of Allergy and \\ Infectious Diseases, National Institutes of \\ Health, Bethesda, MD, 20892, USA; \\ ${ }^{2}$ Liver Transplantation Center, Azienda \\ Ospedaliera Brotzu, Cagliari, Italy; \\ ${ }^{3}$ NIAID Collaborative Bioinformatics \\ Resource, National Institute of Allergy \\ and Infectious Diseases, National \\ Institutes of Health, Bethesda, MD, \\ 20892, USA; ${ }^{4}$ Advanced Biomedical \\ Computational Science, Frederick \\ National Laboratory for Cancer Research \\ Sponsored by the National Cancer \\ Institute, Frederick, MD, 21702, USA
}

Introduction: HCC is the third leading cause of cancer-related death worldwide, with chronic viral hepatitis accounting for more than $70 \%$ of the cases. Therapeutic options are limited and ineffective. The increasing use of immune-based therapies in solid tumors highlights the need to expand our knowledge on the immunologic microenvironment of HCC.

Methods: Access to liver samples from 20 well-characterized patients with HCC associated with $\operatorname{HCV}(n=9)$ or HBV $(n=11)$ gave us the opportunity to study the immunologic landscape in these tumors. For each patient, RNA-sequencing was performed on the tumor and surrounding nontumorous tissue.

Results: We found that both HCV- and HBV-HCC are associated with a predominance of downregulated genes (74\% and 67\%, respectively). Analysis of the immune landscape using a curated gene list showed 216 of 2481 (9\%) immune genes in HCV-HCC and 164 of 2560 (6\%) in HBV-HCC. However, only 8 immune genes (4\%) were upregulated in HCV-HCC and $27(16.5 \%)$ in HBV-HCC. HCV-HCC was characterized by an enrichment of downregulated genes related to T-cell activation and oxidative stress. The dramatic downregulation of immune genes related to T-cell activation in HCV-HCC prompted us to perform an extensive immunohistochemistry analysis on paraffin-embedded liver specimen. Interestingly, we found a significant reduction of immune-cell infiltration (CD3, CD8 and CD20 positive cells) within the tumor. Moreover, we observed that HCV-HCC is characterized by an enrichment of M2-like CD68-positive cells. These data are consistent with the dramatic downregulation of immune-cell infiltration seen in HCV-HCC. Conversely, HBV$\mathrm{HCC}$ was characterized by upregulation of genes related to monocyte/macrophage activation and cell cycle control, and downregulation of genes involved in various cell metabolisms.

Conclusion: This study demonstrates a distinctive molecular signature and immune landscape in HCC of different viral etiology, which could provide new insights into pathogenesis and lead to the development of novel immune-based therapies.

Keywords: hepatocellular carcinoma, hepatitis C virus, hepatitis B virus, RNA-sequencing, molecular signature, immune landscape

\section{Introduction}

Hepatocellular carcinoma (HCC) is the sixth most common cancer and the third leading cause of cancer-related death worldwide. ${ }^{1,2}$ The incidence is increasing worldwide, with an estimated global incidence rate of 9.3 per 100,000 person-year in 2018 and a corresponding mortality rate of $8.5 .^{2}$ Thus, the incidence and
Hepatic Pathogenesis Section, Laboratory of Infectious Diseases, National Institute of Allergy and Infectious Diseases, National Institutes of Health, Bethesda, MD, 20892, USA

Tel + I 240-669-592I

Fax + I 301-402-0524

Email pfarci@niaid.nih.gov 
mortality of HCC are roughly equivalent. Moreover, HCC has a poor 5-year survival, ${ }^{1}$ with a dramatic increase in mortality over the past decades in contrast with the decreasing mortality reported for several tumors. ${ }^{3}$ Although the pathogenesis of $\mathrm{HCC}$ remains to be elucidated, chronic infection with hepatitis $\mathrm{C}$ virus (HCV) and hepatitis B virus (HBV) account for $71 \%$ of all HCC cases worldwide. $^{4,5}$ Both of these viruses are included in the list of carcinogenic viruses. ${ }^{6}$ Cirrhosis is the single most important risk factor for $\mathrm{HCC}$, being present in more than $80 \%$ of the cases. ${ }^{7}$ The use of long-term antiviral therapy in patients with chronic hepatitis B and the success of direct-acting antivirals in eradicating HCV in over $95 \%$ of patients have significantly decreased but not eliminated the risk of $\mathrm{HCC}^{8}$

$\mathrm{HCV}$ is an enveloped RNA virus belonging to the Flaviviridae family. ${ }^{9} \mathrm{HCV}$ does not integrate into the host genome, and therefore it likely promotes hepatocarcinogenesis through chronic inflammation, liver regeneration and fibrosis rather than through a direct oncogenic effect. $^{10,11}$ However, experiments in mouse models suggested that overexpression of the $\mathrm{HCV}$ core protein may promote hepatocarcinogenesis. ${ }^{12} \mathrm{HBV}$ was the first virus associated with the development of $\mathrm{HCC}^{13}$ and is the leading cause of HCC worldwide. ${ }^{5}$ Although the availability of an effective vaccine against HBV holds promise for the elimination of $\mathrm{HBV}$-associated HCC, more than 257 million chronic HBsAg carriers in the world are still at increased risk of developing cirrhosis and HCC. ${ }^{14} \mathrm{Of}$ note, approximately $20 \%$ of HBV-related HCC cases do not present with cirrhosis, suggesting a direct oncogenic effect of HBV. ${ }^{15} \mathrm{HBV}$ is the prototype member of the Hepadnaviridae family. It contains in its interior a nucleocapsid with the viral genome, a $3.2 \mathrm{~Kb}$ relaxed circular double-stranded DNA (dsDNA). After entry into hepatocytes, the nucleocapsid is released into the cytoplasm and migrates to the nucleus, ${ }^{16}$ where the viral genome can integrate into the host DNA, causing insertional mutagenesis and activation of cancer driver genes. $^{17}$

$\mathrm{HCC}$ is one of the most heterogenous cancers comprising distinct molecular and clinical subgroups. ${ }^{18,19}$ Interestingly, it has been shown that only about $20 \%$ of HCC cases, regardless of the tumor etiology, show a high to moderate levels of immune cell infiltration while the vast majority displays a low degree of immune-cell infiltration. $^{18,20,21}$ The immune microenvironment of $\mathrm{HCC}$ has been studied in recent years, ${ }^{20-24}$ but there is limited information on whether the immune-cell infiltration varies according to the different viral etiology. Given the involvement of the immune system in the pathogenesis of chronic viral hepatitis ${ }^{22-24}$ and the increasing use of immune-targeted therapies for solid tumors, it is important to investigate the immune landscape of HCC in order to identify differences and similarities among HCCs of different viral etiology. Access to a unique collection of paired liver specimens (tumor and surrounding nontumorous tissue) from 20 well-characterized Caucasian patients with HCV- and HBV-HCC, seen at a single center in Italy, gave us the unique opportunity to define the molecular signature and the immunologic landscape of these tumors to better understand their differences and similarities.

\section{Materials and Methods}

\section{Patients}

The study included liver specimens from 9 patients with $\mathrm{HCV}$-associated HCC, 1 female and 8 males, aged $60 \pm 8$ years (mean $\pm \mathrm{SD}$ ), and 11 patients with HBV-associated HCC, 1 female and 10 males, aged $57.7 \pm 7.7$ years. The clinical, virologic, and histopathological features of the patients were previously reported. ${ }^{25,26}$ All patients were followed at the Liver Transplantation Center of the Brotzu Hospital in Cagliari, Italy. Informed consent was obtained from all subjects involved in the study. The protocol was approved by the ethical Committee of the Hospital Brotzu (Cagliari, Italy), and by the Office of Human Subjects Research of the NIH, granted on the condition that all samples were deidentified.

\section{RNA-Sequencing}

Total RNA from liver tissue was extracted from frozen liver specimens as described previously ${ }^{27}$ using TRIzol reagent (Invitrogen) according to the manufacturer's recommendations. Total RNA quality and integrity were assessed using the Agilent 2100 Bioanalyzer. The mRNA libraries were constructed from 0.5 to $1 \mu \mathrm{g}$ mRNA using the Illumina TruSeq RNA Sample Prep Kits, version 2. The resulting cDNA was fragmented using a Covaris E210. Library amplification was performed using 10 cycles to minimize the risk of overamplification. Unique barcode adapters were applied to each library. Libraries were pooled in equimolar ratio and sequenced together on a HiSeq 2500 with ver 4 flow cells and sequencing reagents. At least 47 million 125 -base read pairs were generated for each individual 
library. Data were processed using RTA 1.18 .64 and CASAVA 1.8.2.

All RNA-sequencing (RNA-seq) samples had a raw yield of at least 60 million paired-end 125 base-pair reads. Data were processed using the Pipeliner workflow (https://github.com/CCBR/Pipeliner). Reads were trimmed to remove contaminating adapter sequences and lowquality bases using cutadapt ${ }^{28}$ and aligned to the human hg38 reference genome and Gencode release 28 using STAR v2.5.2b run in 2-pass mode. ${ }^{29}$ RSEM v1.3.0 ${ }^{30}$ was used for gene-level expression quantification, and limma v 3.40.2 $2^{31}$ was used for voom quantile normalization and paired differential expression analysis. Only genes passing the following cutoff were used for downstream analyses: more than 0.5 counts per million across at least nine or ten samples for HBV and HCV respectively. Selection criteria for transcriptomic analysis require genes to have foldchange greater than +1.0 or lower than -1.0 , and FDRadjusted $\mathrm{P}$ value less than 0.05 . RNA-seq data have been deposited in the NCBI Sequence Read Archive (SRA) (accession number PRNJA719288).

\section{Functional Immune Categories}

We first selected the genes involved in the immune response from the bulk gene expression data (expressed as fold change between tumor and non-tumor tissue) based on a curated list of 828 genes encompassing cell surface markers, secreted proteins, and intracellular factors. This list was generated by combining gene ontology with two databases, Reactome ${ }^{32}$ and Ingenuity [QIAGEN Inc.]. To identify pathways among the differentially expressed immune genes, we utilized the WEB-based Gene SeT AnaLysis Toolkit (WebGestalt, version 2019) analysis using our curated list of immune genes as reference. ${ }^{33} \mathrm{In}$ this web application, we selected the overrepresentation enrichment analysis (ORA), followed by pathway analysis using Reactome as database. ${ }^{32}$ We selected a multiple test adjustment with Benjamini \& Hochberg (BH) correction and set a P-value $<0.05$.

\section{Immunohistochemistry}

Immunohistochemical staining of formalin fixed paraffinembedded liver sections was performed using a panel of antibodies including CD3, CD8, CD20, CD68 (Dako), alpha-SMA (Abcam), and CD163 (Novus Biologicals). Briefly, sections of 3 to $5 \mu \mathrm{m}$ were deparaffinized through graded alcohols and xylene. Immunohistochemical staining was performed after antigen retrieval using either citrate buffer (10 mmol, pH 6.0) or EDTA (1 mmol, pH 9.0). Slides were incubated in Tris-goat serum (3\%) for 15 min and then incubated at room temperature with primary antibodies. Detection was carried out on the automated system BenchMark XT autostainer (Ventana Medical Systems) according to the manufacturer's instructions. Images were taken using an Olympus BX41 microscope, objective UPlanFI $20 \times / 0.75$, with an adaptor U-TV0.5xC using a digital camera Q-imaging Micropublisher 5.0 RTV. The images were captured using Q-Capture version 3.1.

\section{Statistical Analysis}

Pathway analysis was performed using Ingenuity Pathway Analysis (IPA, version 01-19-00, Qiagen Redwood City, www.qiagen.com/ingenuity). The association of genes to pathways was evaluated as the ratio between the number of genes present in the dataset and the total number of genes that map to the same pathway. The Fisher's exact test was also used to calculate the probability of such association. The chi-square test was used to analyze the frequency of the differentially expressed genes in one immune category (T-cell immune response) between HCVHCC and HBV-HCC. Prism version 8.0.1 (GraphPad software) was used for graphical representation of the data and statistical analysis. Statistical significance was set at $\mathrm{P}<0.05$.

\section{Results}

\section{Characteristics of the Patients}

RNA-sequencing (RNA-seq) was performed in a cohort of 20 well-characterized patients with viral-associated HCC, which included 9 patients with $\mathrm{HCV}$ and 11 patients with HBV-associated HCC. For each patient, liver specimens obtained from the tumor and surrounding nontumorous tissue were analyzed. The demographic, clinical, serological, and pathological features of the 9 patients with $\mathrm{HCV}^{25,34}$ and the 11 with $\mathrm{HBV}^{26}$ have previously been reported. In the surrounding nontumorous tissue, cirrhosis was documented in $90 \%$ of $\mathrm{HCV}$-associated $\mathrm{HCC}$ patients $^{25}$ and in $82 \%$ of HBV-associated HCC. The tumor size was less or equal to $3 \mathrm{~cm}$ in 5 patients and larger than $3 \mathrm{~cm}$ in the remaining 4 patients with HCV$\mathrm{HCC} ;{ }^{25}$ the grade of the tumor differentiation was found to be G2 in 5 patients and G3 in the remaining 4 patients. ${ }^{25}$ All patients were positive for HCV RNA and most had genotype 1 (89\%). ${ }^{34}$ In HBV-HCC, the tumor size was between 2 and $3 \mathrm{~cm}$ in 8 patients and larger than $3 \mathrm{~cm}$ in 
the remaining 3 patients; ${ }^{26}$ the grade of tumor differentiation was G2 in 7 patients, G3 in 3 patients, and G4 in the remaining patient. ${ }^{26}$ All patients were HBsAg positive and anti-HBe positive with very low levels of HBV replication since all patients were under nucleos(t)ide therapy. ${ }^{26}$

\section{Pathway Analysis in Tumor versus Nontumorous Liver Tissue in HCV- and HBV-Associated HCC}

For each of the 20 patients studied, RNA-seq was performed using RNA obtained from paired tumor and nontumorous liver specimens. The results of these analysis showed that both HBV- and HCV-associated tumors are characterized by a predominant gene downregulation (Figure 1), confirming previous data that we reported using microarray gene expression profiling. ${ }^{25,26}$ In HCVHCC (Figure 1A), we detected 2481 differentially expressed genes within the tumor with a large preponderance of them downregulated (1831; 74\%). A complete list of the differentially expressed genes in $\mathrm{HCV} \mathrm{HCC}$ is reported in Table S1. Of the 20 top-scored pathways in HCV-HCC, the top-scored was represented by hepatic fibrosis and hepatic stellate cell activation, with only 1 gene upregulated and 48 genes downregulated (Figure 1B). However, the most peculiar feature of HCVHCC was a group of 14 pathways involved in the regulation of the immune response, with $95 \%$ downregulated genes (Figure 1B). These pathways encompassed genes of both the innate and adaptive immune responses, although the $\mathrm{T}$ cell-mediated immune response was the most represented (Th1 and Th2 activation pathway, communication between innate and adaptive immune cells, Th1 pathway, primary immunodeficiency signaling, Th2 pathway, hematopoiesis from pluripotent stem cells, and dendritic cell maturation). The remaining 5 pathways were not directly related to the immune system; two of them were associated with lipid metabolism (LXR/RXR activation and eicosanoid signaling) and three were related to signal transduction of G-protein coupled receptor (sperm motility, G-protein coupled receptor signaling and cAMPmediated signaling) (Figure 1B).

In HBV-HCC, the analysis identified 2560 differentially expressed genes with a high prevalence of downregulated genes within the tumor $(1712 ; 67 \%)$, although there was a higher proportion of upregulated genes as compared with HCV-HCC (848; 33\%) (Figure 1C). A complete list of the differentially expressed genes in HBV HCC is reported in
Table S2. When we analyzed the 20 top-scored pathways, we found that 5 pathways were in common with HCV-HCC (hepatic fibrosis and hepatic stellate cell activation, primary immunodeficiency signaling, LXR/RXR activation, acute phase response signaling, and complement system) (Figure 2). The presence of the hepatic fibrosis and hepatic stellate cell activation pathway with the vast majority of genes downregulated (98\% in HCV-HCC and $84 \%$ in HBVHCC) suggests that in HCC the extracellular matrix production was inhibited regardless of the tumor etiology, a finding previously reported by our group using microarray assay. ${ }^{35}$ The other 4 pathways shared by HCV- and HBV-HCC included LXR/RXR activation, which is related to lipid metabolism, and 3 pathways related to the immune response, specifically B-cell activation and antibody production (primary immunodeficiency signaling) and acute phase response (acute phase response signaling and complement system) (Figure 2). In contrast to HCV-HCC, whose signature was characterized by a prominent dysregulation of genes involved in the T-cell immune response, in HBV-HCC we did not find any pathway related to T-cell immune activation and regulation (Figure 1D). The most peculiar feature of HBV-HCC was the presence of 9 pathways associated with the regulation of cell metabolism, which were uniquely detected in HBV-HCC (Figures 1D and 2). These included 3 pathways related to the family of nuclear receptors, which play a central role in the regulation of lipid metabolism (LPS/IL-1 mediated inhibition of RXR function, FXR/ RXR activation, LXR/RXR activation, and PXR/RXR activation). The other 6 pathways were involved in the degradation of several substrates, such as acetone, bupropion, tyrosine, histidine, nicotine and $\alpha$-tocopherol (Figure 1D). The remaining two pathways were related to hepatic synthesis (estrogen biosynthesis and coagulation system). Although these pathways were all characterized by the prevalence of downregulated genes, in HBV-HCC we uniquely detected 4 pathways involved in cell cycle regulation and control (estrogen-mediated S-phase entry, GADD45 signaling, cyclins and cell cycle regulation, and cell cycle control of chromosomal replication) with the vast majority (80\%) of genes upregulated (Figure 1D).

\section{Analysis of the Immune Microenvironment in Viral-Associated $\mathrm{HCC}$}

Given the significant presence of pathways related to the immune response in $\mathrm{HCV}-\mathrm{HCC}$, we further investigated 
A

A $\mathrm{HCV}-\mathrm{HCC}$

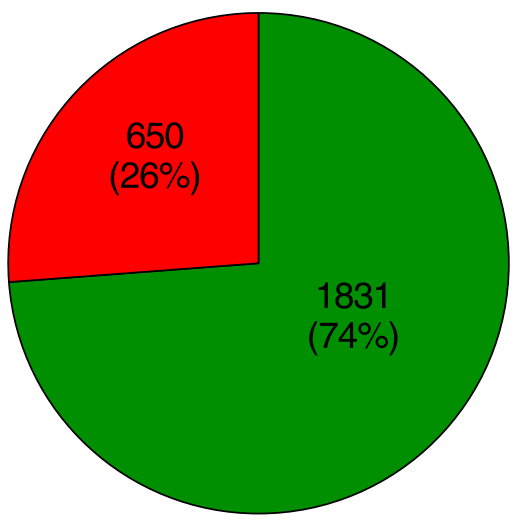

$n=2481$

c

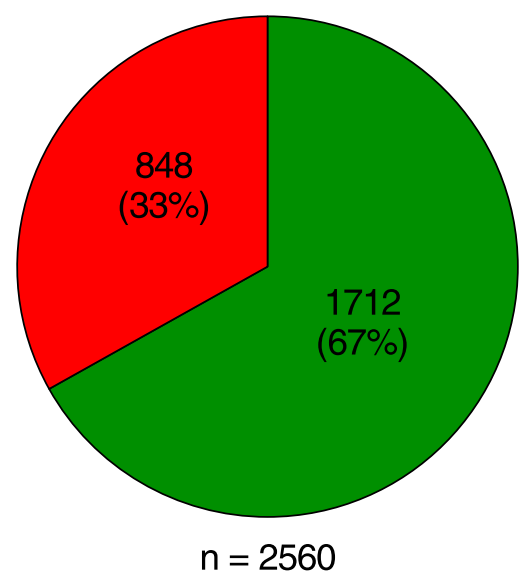

B

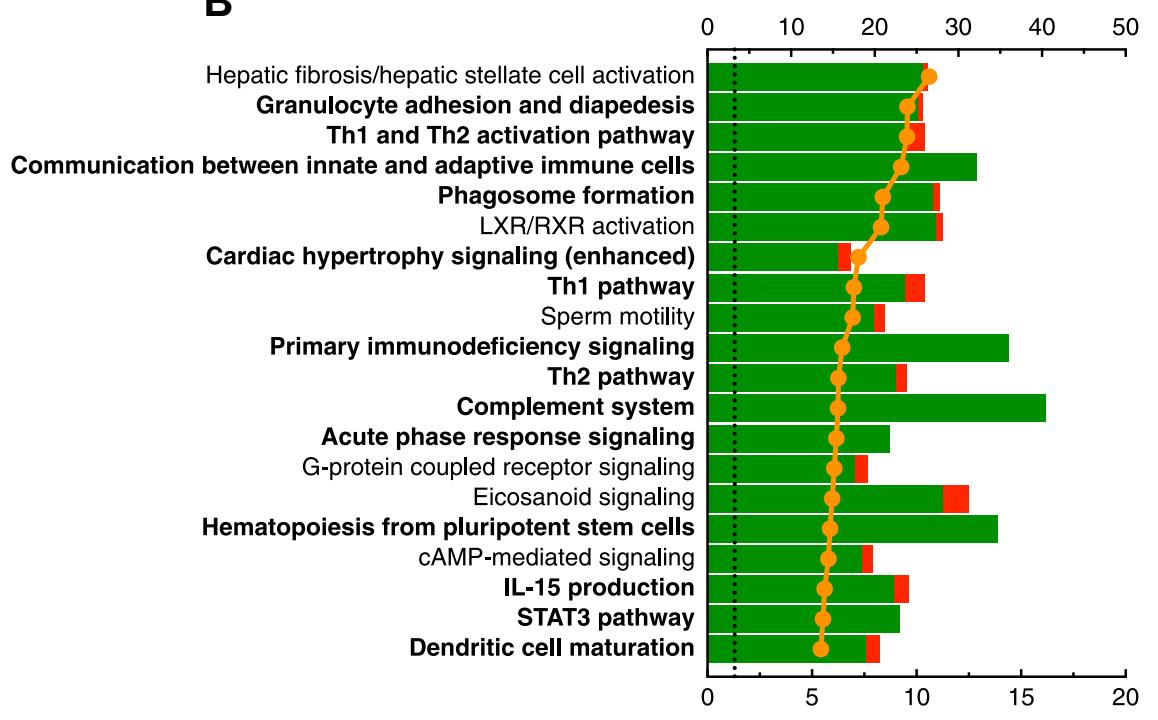

D

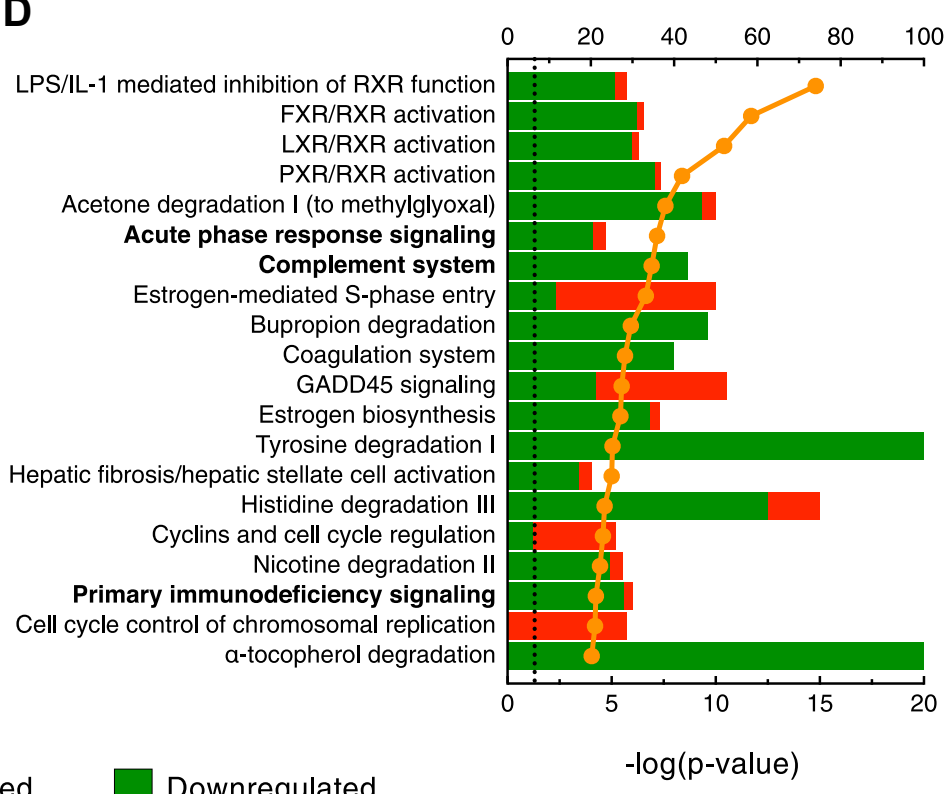

Upregulated $\square$ Downregulated
Percentage of downregulated and upregulated genes

Figure I Molecular signature of HCV- and HBV-associated HCC. Pie charts showing the number of upregulated and downregulated genes (A) and the top scored canonical pathways (B) in HCV-HCC. Pie charts showing the number of upregulated and downregulated genes (C) and the top scored canonical pathways (D) in HBV-HCC. Columns (quoted on the top axes) represent the percent ratio between the number of genes present in the dataset and the total number of genes present in the database, for each pathway. The green and red portions of columns indicate downregulated and upregulated genes, respectively. The orange line (quoted on the bottom axes) shows the statistical significance of each biological process, expressed as the negative p-value of Fisher exact test. The dotted line indicates the significance threshold corresponding to $\mathrm{P}=0.05$ on the log scale. The pathways in bold indicate those associated with the immune system. Pathway analysis was derived from Ingenuity Pathway Analysis (http:// www.ingenuity.com).

the immune landscape of this tumor and compared it with that of HBV-HCC. First, we selected all genes involved in the immune response from the bulk of gene expression data (expressed as fold change between tumor and nontumor tissue) using a curated list of 828 genes that we generated combining gene ontology with two databases,
Reactome $^{32}$ and Ingenuity [QIAGEN Inc.], which encompass genes related to cell surface markers, secreted proteins, and intracellular factors.

In HCV-HCC, 216 of 2481 (9\%) genes were related to the immune response, and only a minority of them was upregulated ( 8 of $216 ; 4 \%$ ) (Figure $3 \mathrm{~A}$ ). HBV- 


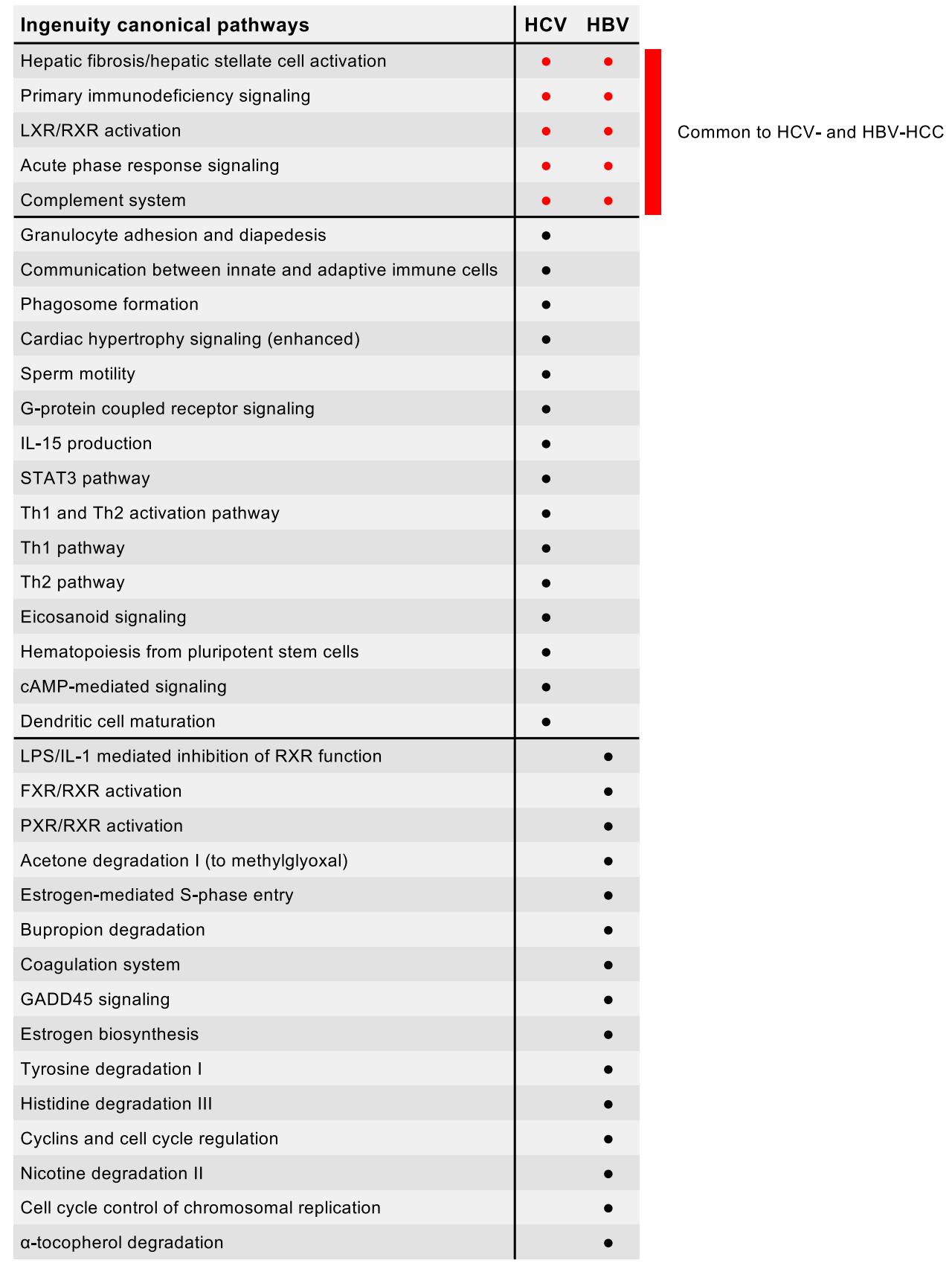

Figure 2 Synopsis of the 20 top-scored pathways using all $248 \mathrm{I}$ genes differentially expressed in HCV-HCC and all 2560 genes from HBV-HCC obtained from paired liver specimens (tumor and nontumorous tissue for each patient). The common pathways are listed in the first rows, shown by red dots.

HCC showed a lower proportion of differentially expressed genes related to the immune response (164 of 2560 genes; $6 \%$ ) but a higher number of upregulated genes (27 of $164 ; 16.5 \%$ ) compared with HCV-HCC; the remaining immune genes were downregulated (137 genes; 83.5\%) (Figure 3B). All the differentially expressed immune genes are listed in Table S3 and S4.
Next, we analyzed the pathways among the immune genes by using the Reactome database as reference. ${ }^{32}$ This analysis showed marked differences between HCV-HCC and HBV-HCC. Among the 15 significant pathways identified in HCV-HCC, we found 3 pathways related to the T-cell immune response (cross-presentation of particulate exogenous antigens, generation of second messenger 
A

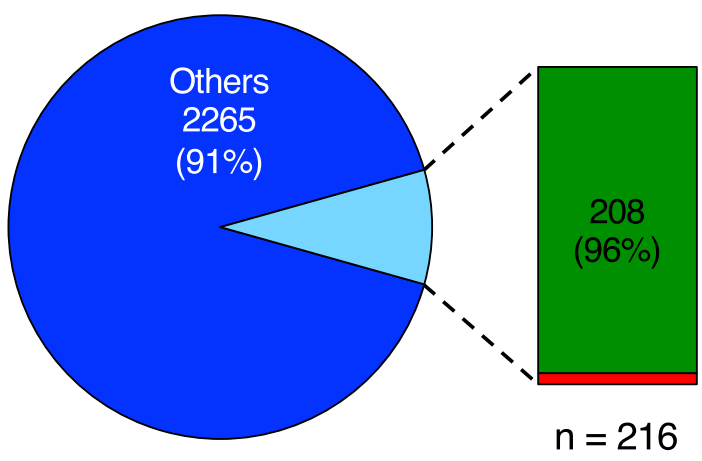

$(9 \%)$
B

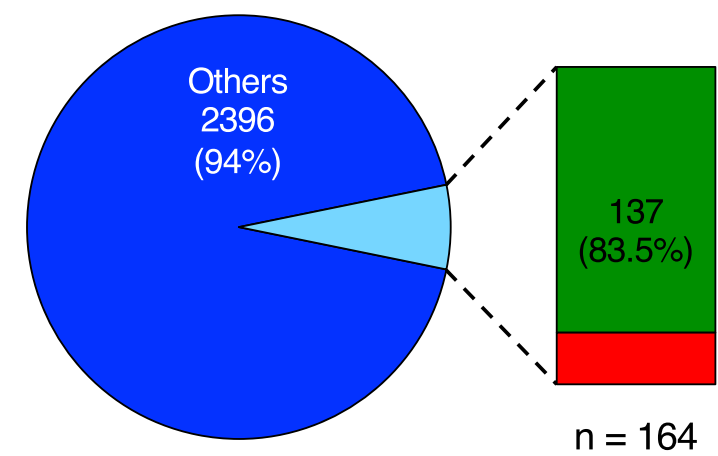

$(6 \%)$

Immune genes

Upregulated

Downregulated

Figure 3 Proportion of immune genes in HCV- and HBV-associated HCC. Graphs showing the proportion of immune genes (light blue) on the total differentially expressed genes (pie charts) and the number of downregulated and upregulated immune genes (vertical charts) identified in liver tissue of HCV- (A) and HBV-associated HCC (B).

molecules, and PD-1 signaling), with all genes downregulated (Figure 4A). Moreover, we identified a large number of genes not included in these 3 molecular pathways, but functionally related to $\mathrm{T}$ cell-activation and regulation, comprising CD27, CD5L, CD69, CD7, CD86, CD8A, CD8B, CD96, CLEC4G, GATA3, HAVCR2, NFATC1, NFATC2, TAGAP, TIGIT, TIMD4, VSIG4, VTCN1 (Table $\underline{\mathrm{S} 3}$. We also found two pathways, namely, interleukin-10 signaling and GPCR downstream signaling, as well as a series of genes that are not included in these two pathways but are related to chemokine and cytokine production and signaling, mainly associated with the differentiation of T cells (eg, CCL14, CXCL14, IL15RA, IL17RE, IL18R1, IL18RAP, IL1RAP, IL1RL1, IL20RA, IL21RA, IL27, $I L 2 R A, I L 2 R B, I L 4 R, I L 7 R, T G F A, T G F B 3$ ) (Figure 4A and Table S3).

In addition to the pathways related to the T-cell immune genes, another significant and distinctive feature of HCV-HCC was the presence of a group of 5 pathways associated with oxidative stress (signaling by Rho GTPases, Rho GTPases activate NADPH oxidases, regulation of TLR by endogenous ligand, Rho GTPase effectors, and detoxification of reactive oxygen species) mainly related to reactive oxygen species (ROS) production, with all genes downregulated (Figure 4A). The remaining 4 pathways were related to the complement system (creation of $\mathrm{C} 4$ and $\mathrm{C} 2$ activators, classical antibody-mediated complement activation, complement cascade and regulation of complement cascade).
In HBV-HCC, only 9 pathways reached statistical significance (Figure 4B). Interestingly, the first three top-scored pathways were related to the complement system and were in common with HCV-HCC (terminal pathway of complement, complement cascade, and regulation of complement cascade), with all genes downregulated (Figure 4B). A fourth pathway related to the complement system (alternative complement activation) was instead uniquely detected in HBV-HCC. Among these pathways, not only the complement components $(C 1 R, C 1 R L, C 3, C 5, C 6, C 7, C 8 A, C 8 B, C 9)$ and factors of the complement (CFD, $C F H, C F I, C F P$, CFHR3) were downregulated, but also the receptors $C 5 A R 1$, which has chemotactic activity, and $C R 1$, which plays a critical role in clearance of complement-opsonized pathogens (Table S4). The remaining pathways were uniquely detected in HBV-HCC and were involved in platelet activation (post-translational modification: synthesis of GPI-anchored proteins, platelet degranulation, and response to elevated platelet cytosolic $\mathrm{Ca}^{2+}$ ) and related to the response of immune cells to tumor antigens (immunoregulatory interactions between a lymphoid and a non-lymphoid cell) (Figure 4B). Finally, among the upregulated immune genes, the majority of differentially expressed genes were involved in monocyte/macrophage activation (eg, MIF, S100A4, S100A10, S100A11) (Table S4).

\section{Unique and Common Immune Genes in Viral-Associated HCC}

To better identify the differences between HCV-HCC and $\mathrm{HBV}-\mathrm{HCC}$, we further analyzed the immune genes 


\section{A HCV-HCC immune genes $(n=216)$}

Percentage of downregulated and upregulated genes

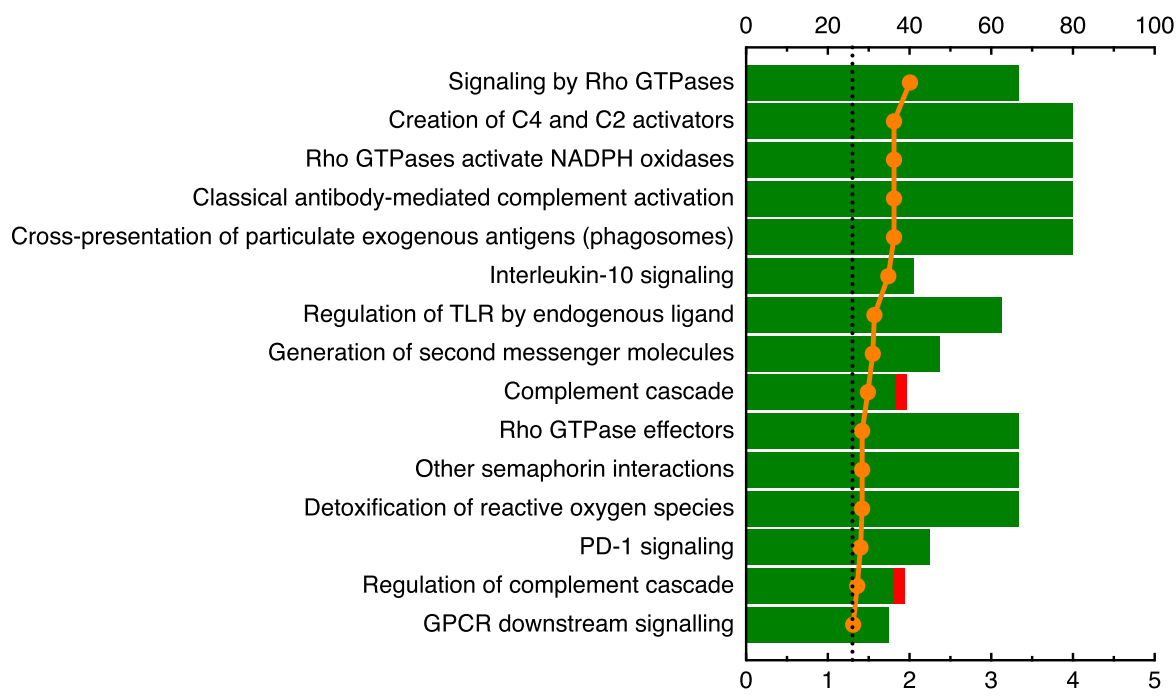

B HBV-HCC immune genes $(n=164)$

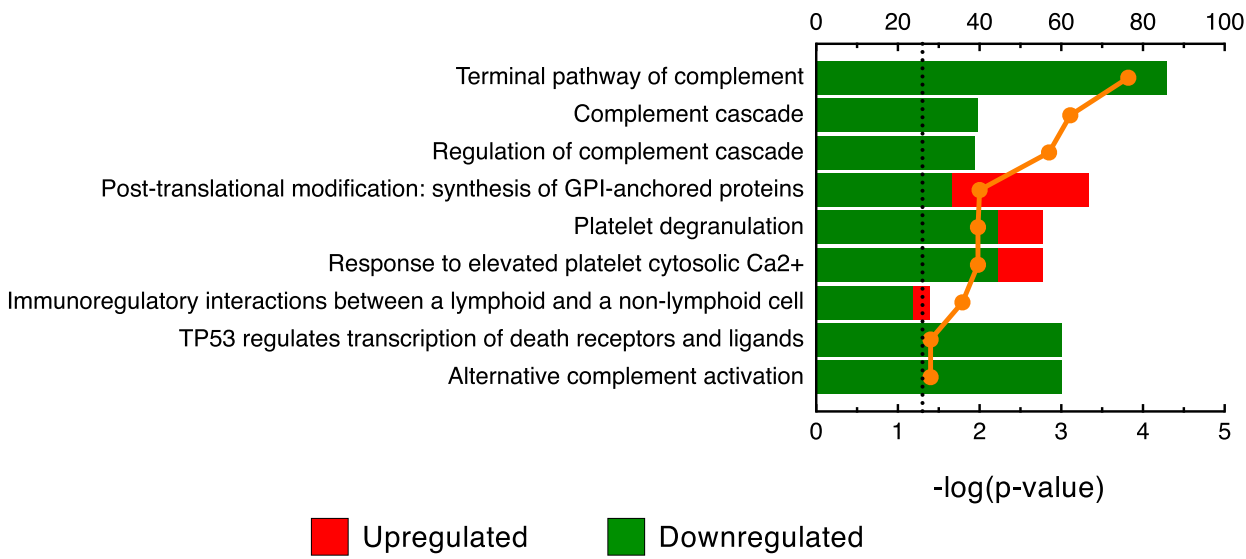

Figure 4 Pathway analysis of the immune genes differentially expressed in HCV- and HBV-associated HCC. Top-scored canonical pathways of immune genes differentially expressed in liver specimens obtained from HCV- $(\mathbf{A})$ and $\mathrm{HBV}$-associated HCC (B). Columns (quoted on the top axes) represent the percent ratio between the number of genes present in the dataset and the total number of genes present in the database, for each biological process. The green and red portions of the columns indicate downregulated and upregulated genes, respectively. The orange line (quoted on the bottom axes) shows the statistical significance of each biological process, expressed as the negative $p$-value of Benjamini \& Hochberg test. The dotted lines indicate the significance threshold corresponding to $P=0.05$ on the log scale. Pathway analysis were obtained by over-representation analysis using our curated gene list as reference and Reactome as pathway database (http://www.webgestalt.org).

uniquely expressed in these two tumors. Our analysis documented that in HCV-HCC 117 of 216 (54\%) immune genes were differentially expressed only in this tumor with the vast majority downregulated (113 of 117 genes; 97\%) (Figure 5A and Table S5). Pathway analysis using the Reactome database as reference ${ }^{32}$ showed that the immune genes uniquely expressed in HCV-HCC were mostly associated with 5 pathways related to T-cell response, in particular T-cell co-stimulation and activation (phosphorylation of CD3 and TCR zeta chains, generation of second messenger molecules, PD-1 signaling, translocation of ZAP-70 to immunological synapse, and IL-10 signaling) (Figure 5B). Among these pathways, we found a downregulation of genes related to antigen presentation (HLA-DPA1, HLA-DPB1, HLA-DQA1, HLA-DRA, HLA$D R B 1)$, cytokine production (CCL2, CCL22, CCR1, CXCL1, CXCL8, IL10RA, IL18, IL1B, ILO), and T-cell activation (CD274, CD3E, CD86) (Table S5). Interestingly, we also found other downregulated genes not included in these pathways, but related to T-cell activation, differentiation, and interaction with other cells (CD53, CD7, CD8B, CD96, GATA3, HAVCR2, TAGAP, 
A HCV-HCC unique genes $\mathrm{n}=117$

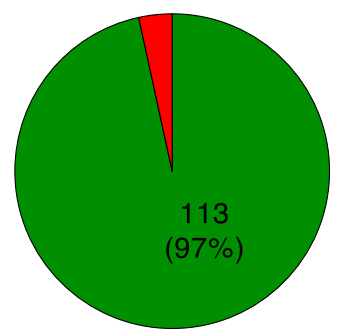

\section{HBV-HCC unique genes}

$$
\mathrm{n}=65
$$

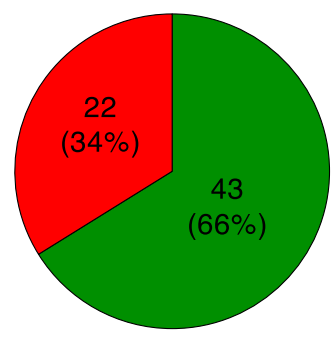

E

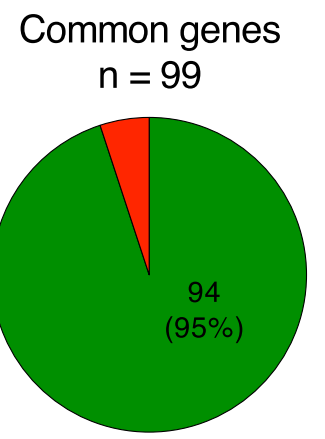

B

Percentage of downregulated and upregulated genes

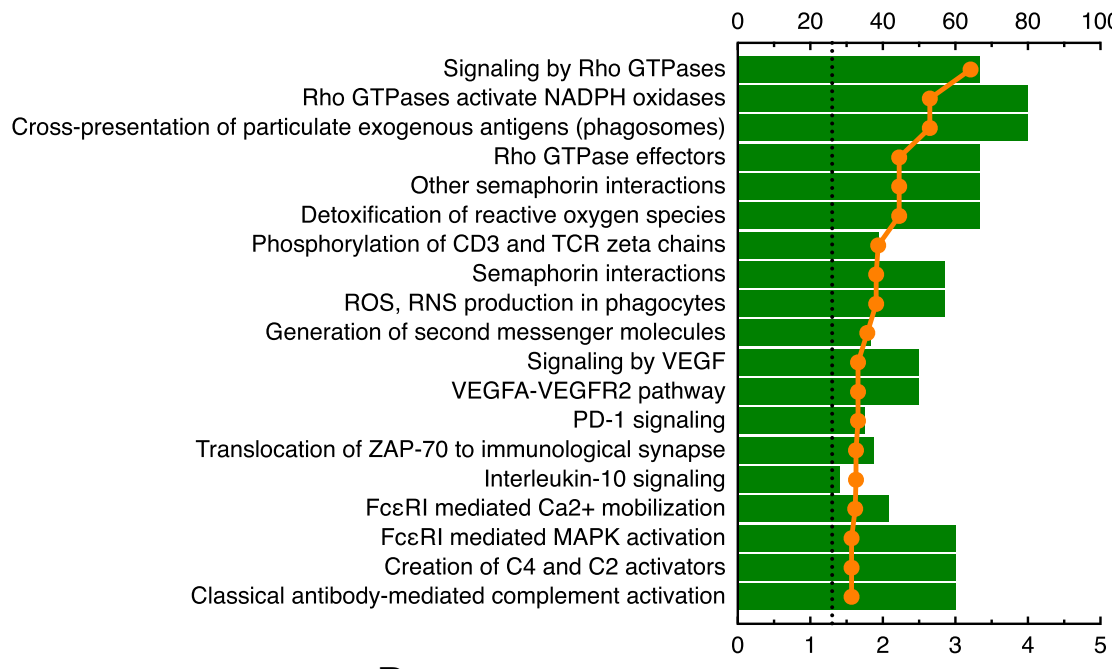

D

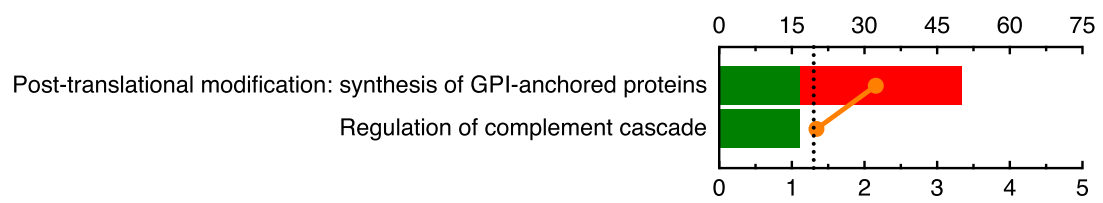

$\mathbf{F}$

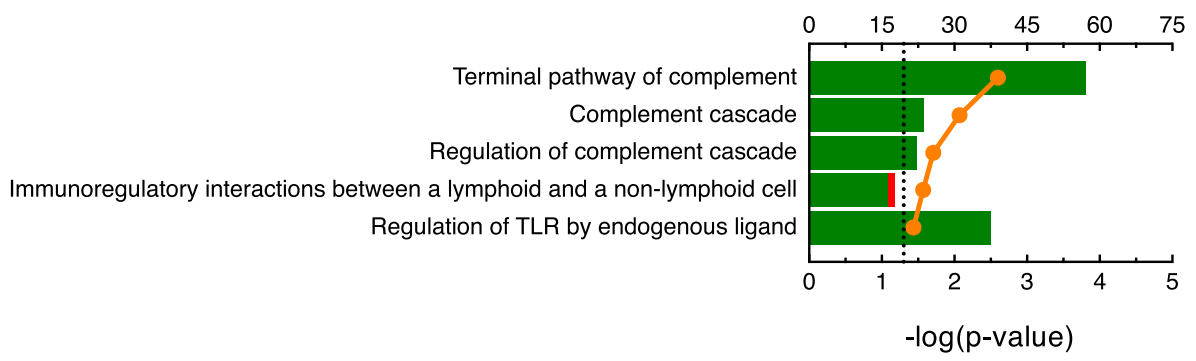

Upregulated Downregulated

Figure 5 Analysis of the immune genes uniquely expressed in HCV- and HBV-associated HCC. Pie charts showing the number of upregulated and downregulated genes (A) and the top-scored canonical pathways (B) in HCV-HCC. Pie charts showing the number of upregulated and downregulated genes (C) and the top scored canonical pathways (D) in HBV-HCC. Pie charts showing the number of upregulated and downregulated genes (E) and the top scored canonical pathways (F) among genes commonly expressed by HCV and HBV. Columns (quoted on the top axes) represent the percent ratio between the number of genes present in the dataset and the total number of genes present in the database, for each biological process. The green and red portions of the columns indicate downregulated and upregulated genes, respectively. The orange line (quoted on the bottom axes) shows the statistical significance of each biological process, expressed as the negative p-value of Benjamini \& Hochberg test. The dotted lines point the significance threshold corresponding to $\mathrm{P}=0.05$ on the log scale. Pathway analysis was obtained by over-representation analysis using our curated gene list as reference and Reactome as pathway database (http://www.webgestalt.org).

VSIG4, VTCN1) (Table S5). The remaining pathways were associated with the innate immune response and oxidative stress (Figure 5B).
In HBV-HCC, 65 of $164(40 \%)$ immune genes were uniquely expressed in this tumor (Figure $5 \mathrm{C}$ ). The proportion of upregulated immune genes in $\mathrm{HBV}-\mathrm{HCC}$ was 
remarkably higher (22 out of $65 ; 34 \%$ ) than in HCV-HCC ( 4 out of $117 ; 3 \%$ ); the remaining 43 immune genes $(66 \%)$ were downregulated (Figure 5C). The list of immune genes uniquely expressed in HBV-HCC is shown in Table S6. We found that only two pathways reached statistical significance (Figure 5D). Among the 43 downregulated genes, there were 16 related to the innate immune response, especially associated with the complement system (eg, C3, C5, C6, CFI, CFH), and 15 associated with the adaptive immune system regulation, in particular related to B-cell regulation (IGHV4-31, FCRL2, HPX, PAX5, FCRLA, KLHL3, BLNK) (Table S6). A few genes were involved in T-cell response regulation ( $L A G 3$, CD160, TIAM1, TRGC2, TBX21). The remaining 12 downregulated genes were involved in cytokine production and signaling (CXCR5, IL13RA2, CXCL2, ILIRN, IL33, CISH, CCL15-CCL14, IFNLR1, TGFBR3, IFITM1, IFNAR1, IL6ST). Among the 22 upregulated genes, 9 were related to the innate immunity (MIF, CTSA, CTSC, TALDO1, S100A11, VNN2, NOS2, ITGA2, LOXL2), 8 were involved in cytokine signaling (TREM2, CCL20, CD109, TRIM45, SQSTM1, CIQTNF3, TNFRSF4, $A P O B E C 3 B)$ and the remaining 5 were related to the B-cell development (IKBKE, SEMA4F, CD34, HMGA1, GIMAP1-GIMAP5) (Table S6).

RNA-seq analysis showed that 99 immune genes were commonly expressed in HBV- and HCV-associated HCC with 94 genes (95\%) downregulated and only 5 genes (5\%) upregulated (Figure 5E). The list of the immune genes commonly expressed in both HCV-HCC and HBV$\mathrm{HCC}$ is shown in Table S7. Interestingly, the 3 top-scored pathways were associated with the complement system with all genes downregulated (terminal pathway of complement, complement cascade, and regulation of complement cascade) (Figure 5F). One pathway was related to the regulation of the lymphoid cell response to self and tumor antigens (immunoregulatory interactions between lymphoid and non-lymphoid cell) and one was associated with the response to damage-associated molecular patterns (regulation of TLR by endogenous ligand) (Figure 5F), both of which are important in the immune response to solid tumors. In particular, genes related to the leukocyte immunoglobulin-like receptors (LILRA1, LILRA2, LILRA5, LILRB1, LILRB5), which play a crucial role in the modulation of several immune cell activity, were downregulated (Table S7). These molecules play an important role as a surveillance system in monitoring the dysregulation of MHC class I presentation during cancer or viral infection. ${ }^{36}$ Moreover, we observed a common downregulation of genes related to B-cell activation (eg, CD79A, FCRL1, FCRL3, FCRL5, MS4A1, MZB1, NFKBIZ, POU2AF1, SELP), and a downregulation of genes related to dendritic cell activation and antigen presentation (eg, CCR7, CDID, IGHG3, IRF8, MICB, PSMD4, SIGLEC7, SIGLEC11, TLR4) (Table S7). Finally, we also detected a strong downregulation of three chemokines (CXCL14, CXCL12, CCL19) involved in immune cell chemoattraction, suggesting a reduced recruitment activity within the tumor microenvironment. Strikingly, all the common genes were similarly downregulated or upregulated in both $\mathrm{HCV}-\mathrm{HCC}$ and HBVHCC with the only exception of S100A6, which was upregulated in HBV-HCC and downregulated in HCVHCC (Table S7).

\section{Immunohistochemistry in HCV-HCC}

To validate the immune profile of HCV-HCC, which was characterized by a dramatic downregulation of T-cell related genes, we performed an extensive immunohistochemistry analysis in paraffin-embedded paired liver specimen from the same patients used for RNA-seq. The presence of CD3 (a marker of T cells), CD8 (a marker of CD8 T cells), CD20 (a marker of B cells), CD68 (a marker of monocyte/macrophage), CD163 (which is associated with M2-like phenotype of macrophages), and $\alpha$-SMA (a marker of activation of hepatic stellate cells) in both tumor and nontumorous liver tissue was examined.

We found that while in all patients with HCV-HCC the surrounding nontumorous tissue was characterized by abundant immune-cell infiltration (CD3, CD8, and CD20) as well as hepatic stellate cell activation ( $\alpha$-SMA), within the tumor there was a significant reduction of immune-cell infiltration with few cells positive for CD3, CD8 and CD20 as well as a strong reduction of hepatic stellate cells activation, as shown by the weak expression of $\alpha$-SMA (Figure 6). Thus, these data are consistent with the dramatic downregulation of immune genes related to T-cell activation seen in patients with HCV-HCC by RNA-seq. Interestingly, in contrast to the lack of T-cell infiltration within the tumor, we found that CD68, a marker of monocytes and macrophages, was expressed both in the tumor and in the surrounding nontumorous tissue (Figure S1). Consistent with this finding, CD68 was not found to be differentially expressed by RNA-seq. To further define the phenotype of these cells, we performed staining with CD163, which showed that the vast majority of CD68 
A
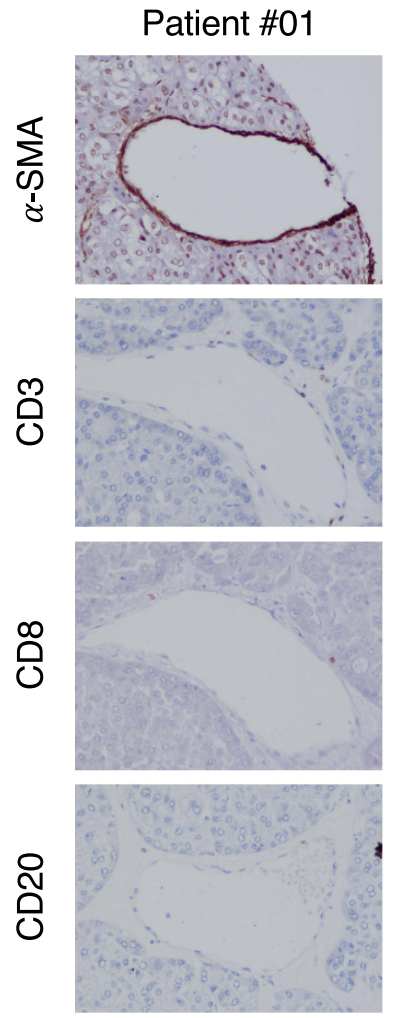

B
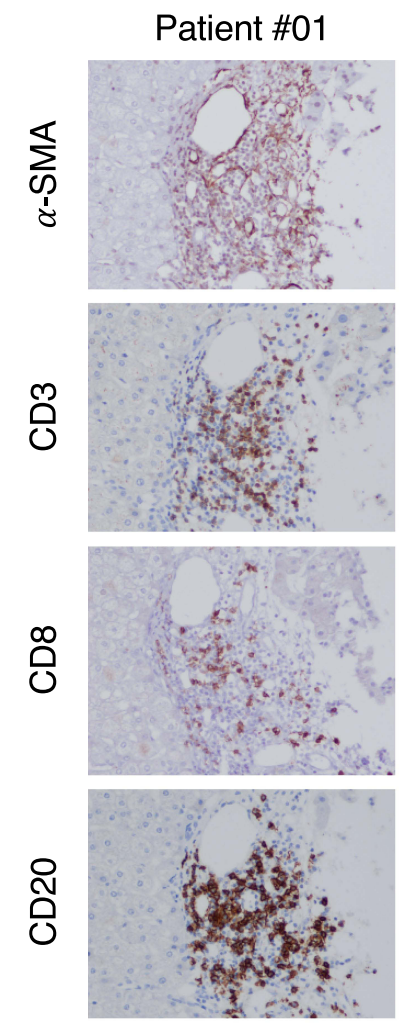

\section{Patient \#02}
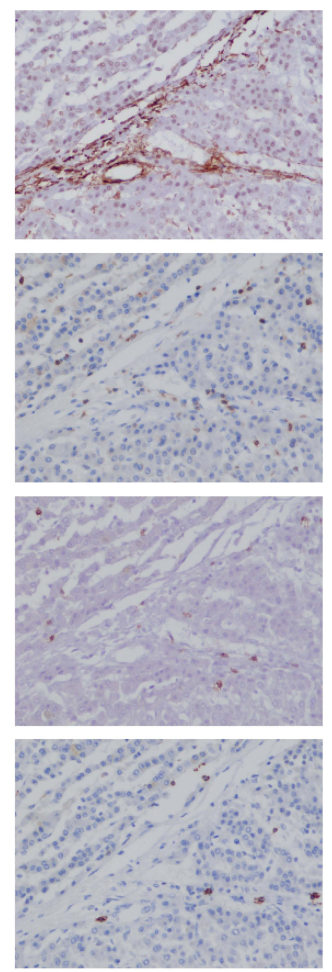

Patient \#02
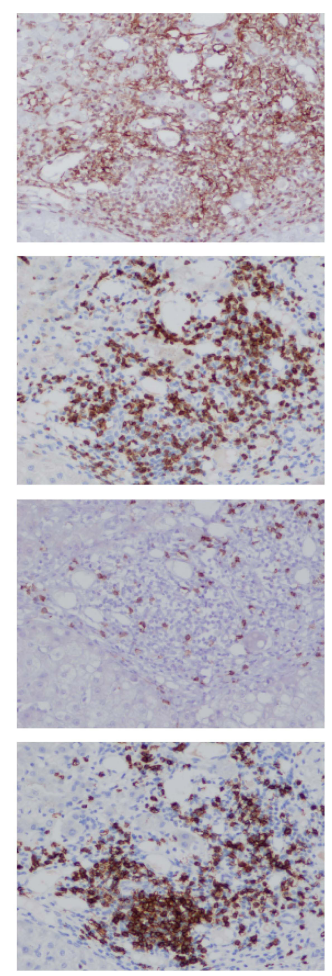

Patient \#03
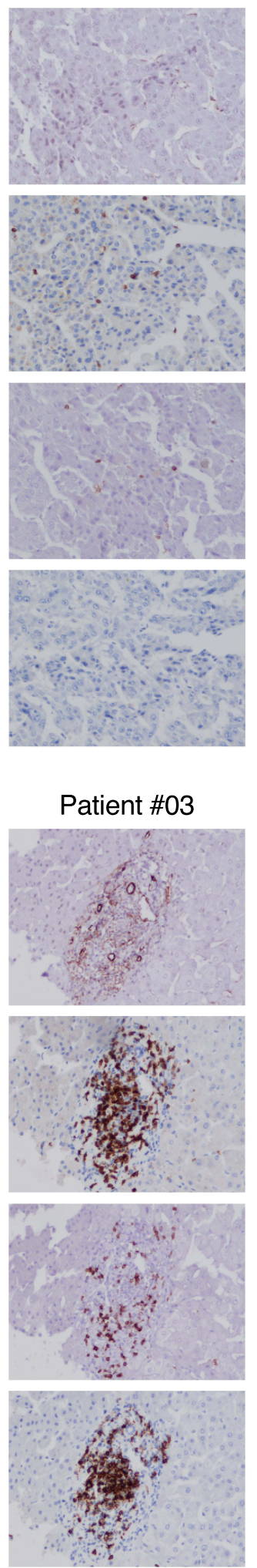

\section{Patient \#04}
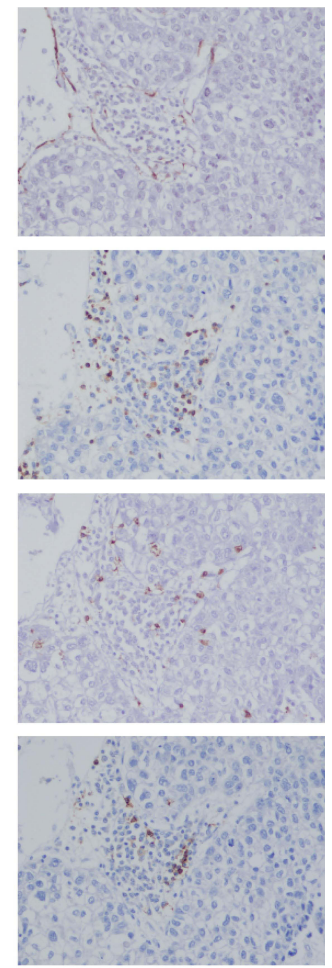

\section{Patient \#04}
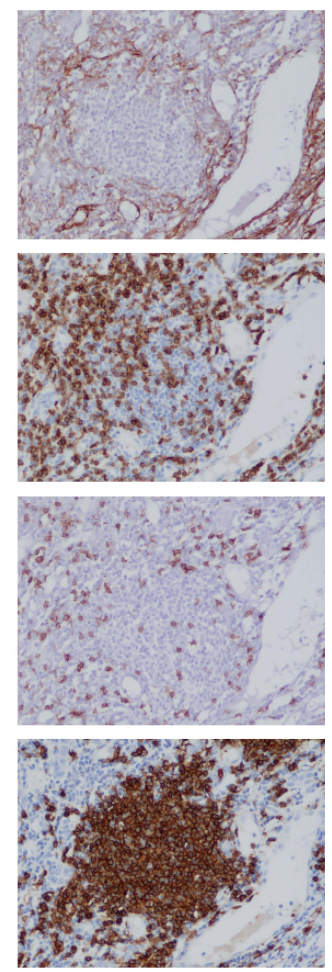

Figure 6 Immunohistochemical staining of immune cell markers in liver tissue from representative patients with HCV-associated HCC. Liver specimens obtained at the time of liver transplantation were stained with monoclonal antibodies against alpha-SMA, CD3, CD8, and CD20 with the use of immunoperoxidase both within the tumor (A) and in the surrounding nontumorous tissue (B) from four representative patients with HCV-HCC. (Magnification: $x 20$ ). 
positive cells also co-expressed CD163, consistent with a M2-like phenotype (Figure 7). Thus, these data support the hypothesis that in HCV-HCC the presence of tumorassociated macrophages (TAM) with a M2-like phenotype may be involved in the suppression of the adaptive immune response against the tumor, as previously described. ${ }^{37}$

\section{Discussion}

Although there is a strong epidemiologic link between chronic infection with HCV or HBV and the development of HCC, ${ }^{4,5}$ the mechanisms whereby these viruses promote hepatocarcinogenesis remain elusive. ${ }^{10,11}$ Taking advantage of a unique collection of liver specimens from well-characterized patients with viralassociated HCC, the aim of this study was to characterize the molecular signature of $\mathrm{HCV}$-associated $\mathrm{HCC}$ using RNA-seq, and to compare it with that of HBVHCC. Our study demonstrated that both tumors are characterized by a predominant gene downregulation, yet distinctive features differentiate $\mathrm{HCV}$ - from $\mathrm{HBV}$ $\mathrm{HCC}$. We found that HCV-HCC is characterized by downregulation of genes related to oxidative stress and T-cell immune response, while HBV-HCC is characterized by upregulation of genes associated with cell cycle control and monocyte/macrophage activation, and downregulation of genes involved in various cell metabolisms
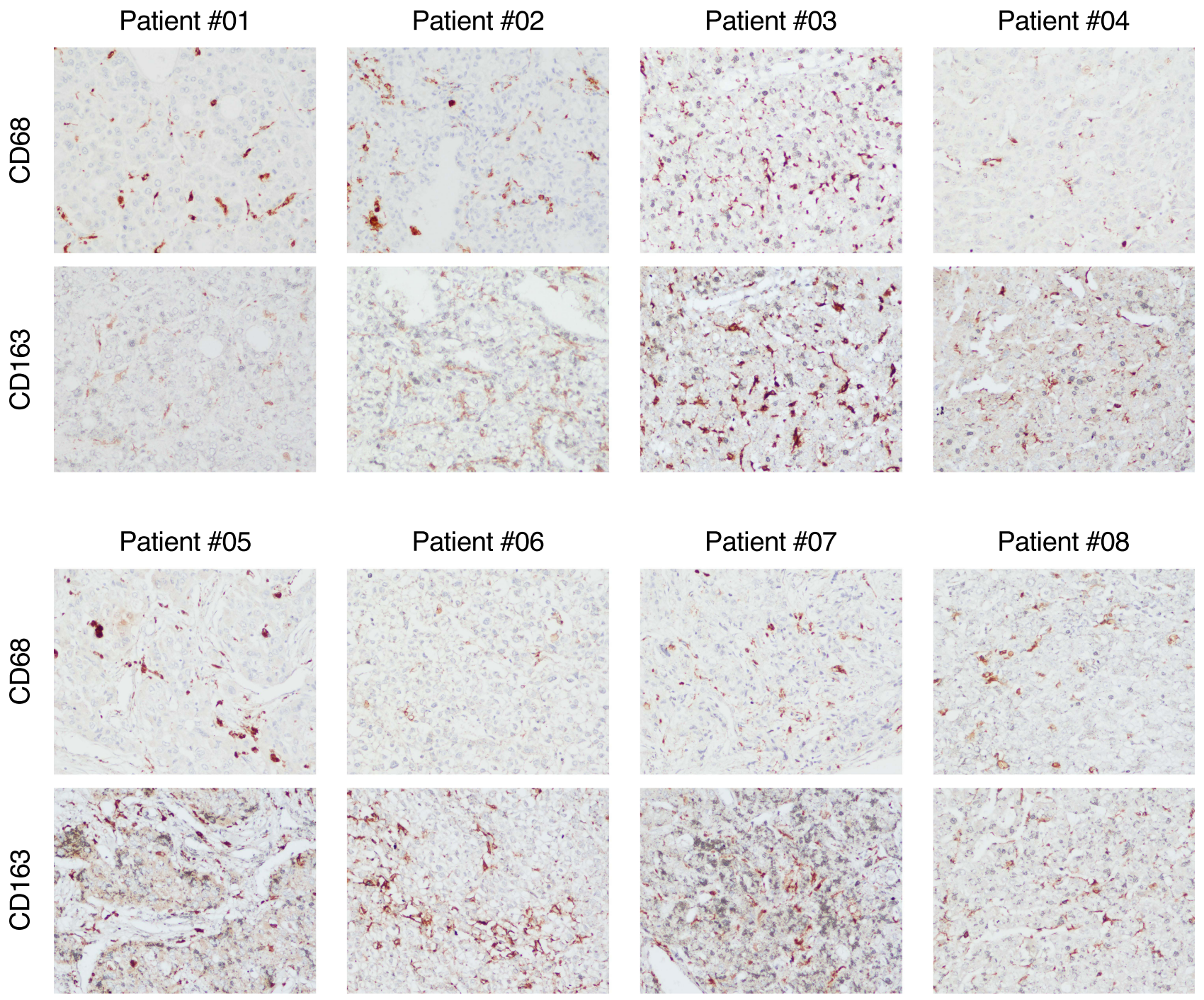

Figure 7 Immunohistochemical staining of a monocyte/macrophage marker CD68 and M2-like phenotype CDI63 in tumor tissue from 8 patients with HCV-associated HCC. Liver specimens obtained at the time of liver transplantation were stained with monoclonal antibodies against CD68 or against CDI63 with the use of immunoperoxidase within the tumor from 8 patients with HCV-HCC. (Magnification: $x 20$ ). 
(Figure 1). Thus, our data collectively suggest that the mechanisms leading to hepatocarcinogenesis differ according to the etiology of the tumor.

$\mathrm{HCV}-\mathrm{HCC}$ was characterized by a significant upregulation of oxidative stress genes in the surrounding nontumorous tissue indicating that necroinflammation and cytotoxic cytokine production may create a sustained proinflammatory environment $^{38}$ leading to iterative hepatocyte damage that may be implicated in the early steps of the hepatocarcinogenesis process. The other distinctive feature of $\mathrm{HCV}-\mathrm{HCC}$ was the presence of a significant number of pathways involved in T-cell immune response, with almost all genes downregulated within the tumor. Further analysis of the immune landscape in the two tumors confirmed an enrichment of genes related to T-cell immune response in $\mathrm{HCV}-\mathrm{HCC}$ as compared with HBV-HCC (chi-square test $\mathrm{p}=0.0493$ ). This gene downregulation was also confirmed by immunohistochemistry, which documented a dramatic reduction of infiltrating immune cells within the tumor as compared with the surrounding nontumorous tissue (Figure 6). However, HCV-HCC was associated with the presence of CD68-positive cells both inside and outside the tumor (Figure S1) with a M2-like phenotype within the tumor (Figure 7). It has been demonstrated that activation of TAM can promote tumor survival and lead to a reduction of the adaptive immune response against the tumor. ${ }^{37}$ Thus, in our study the involvement of TAM in HCV-HCC could contribute to the downregulation of genes related to T-cell immune response and B-cell activation within the tumor, a finding consistent with the lack of tumor T- and B-cells infiltration observed by immunohistochemistry. Another factor that may contribute to the lack of T-cell infiltration within the tumor was the downregulation of important chemokines involved in the recruitment of T cells (eg, CCL2, CCL19, CCL21, CCL22). Interestingly, we observed that 4 genes encoding immune checkpoints, namely, CD86, PD-L1, B7$\mathrm{H} 4$ and TIM-3 were uniquely downregulated in HCV-HCC, while other immune checkpoint genes (eg, CTLA4, LAG3, $P D-1)$ were expressed at similar levels between the tumor and nontumorous tissue. The absence of immune cell infiltration and the downregulation of immune checkpoints are in line with previous studies, which reported the upregulation of immune checkpoints only in a small proportion (20-25\%) of $\mathrm{HCC}$ cases, a finding that was associated with a higher immune cell infiltration within the tumor. ${ }^{18,20,21}$ However, in these studies some virological features, especially the levels of $\mathrm{HCV}$ replication which may influence the immune response within the tumor, were not evaluated. Indeed, it has been shown that the liver microenvironment and the levels of
$\mathrm{HCV}$ replication can influence the activation of CD4 and CD8 $\mathrm{T}$ cells and the expression of several inhibitory receptors. ${ }^{39,40}$ Using the same cohort of patients, we previously demonstrated that only the nontumorous tissue can efficiently sustain HCV replication, while the tumor showed a dramatic reduction in viral replication. ${ }^{34}$ Recently, we reported that the restricted HCV RNA replication within the tumor $^{34}$ was associated with downregulation of tumorassociated calcium signal transducer 2 (TACSTD2), the second most downregulated gene in primary HCC tissue, which is critical for maintaining the proper cellular localization of CLDN1 and OCLN, two major HCV-entry cofactors. ${ }^{25}$

In contrast to $\mathrm{HCV}-\mathrm{HCC}$, we did not find any pathway related to T-cell response in HBV-HCC. We found only two pathways related to the innate immune response, which were mostly associated with the production of acute-phase response proteins from hepatocytes (acute-phase response signaling and complement system), and one pathway related to B-cell activation (primary immunodeficiency signaling), with most of the genes being downregulated. Moreover, we identified a few genes uniquely upregulated in HBV-HCC related to the innate immunity (eg, MIF, NOS2, S100A6, S100A11, TALDO1) suggesting a monocyte/macrophage activation in this tumor, which was not detected in HCVHCC. Another distinctive feature of HBV-HCC was the presence of 4 pathways involved in cell cycle, with the vast majority of genes (80\%) upregulated. Unlike $\mathrm{HCV}$, which does not integrate into the host genome, ${ }^{9}$ the HBV genome can be integrated in infected cells and may contribute to HCC development through the cis- or transactivation of cancer driver genes. ${ }^{16,17}$

\section{Conclusion}

Although the number of patients included in this study was relatively low, they were well characterized in terms of clinical, virological and histopathological features, and they were all seen at a single center in Italy. This homogeneous cohort of Caucasian patients allowed us to perform a reliable comparison of the molecular profiles of HCC of different viral etiology. Our study demonstrated that $\mathrm{HCV}-\mathrm{HCC}$ is characterized by a distinctive molecular signature as compared with HBVHCC, with downregulation of immune genes within the tumor, especially T-cell related genes, and upregulation of oxidative stress genes outside the tumor, probably related to the sustained necroinflammatory environment associated with hepatocarcinogenesis. In contrast, the molecular signature of HBVHCC was characterized by upregulation of genes related to cell cycle control and monocyte/macrophage activation, 
indicating a greater involvement of the innate immune system in this tumor. Additional work on large cohort of patients will be important to confirm the molecular signature and immune landscape in HCC of different etiology, which could provide new insights into the mechanisms of hepatocarcinogenesis and open new avenues for specific immune-based therapies to reduce the ominous prognosis of $\mathrm{HCC}$.

\section{Ethical Approval and Informed Consent}

The study was conducted according to the guidelines of the Declaration of Helsinki and approved by the ethical Committee of the Hospital Brotzu (Cagliari, Italy), and by the Office of Human Subjects Research of the NIH, granted on the condition that all samples were deidentified.

All liver specimens were donated voluntarily with written informed consent and conducted in accordance with the Declaration of Istanbul.

\section{Acknowledgments}

This research was supported by the Intramural Programs of the Division of Intramural Research of the National Institute of Allergy and Infectious Diseases, and Frederick National Laboratory for Cancer Research sponsored by the National Cancer Institute, National Institutes of Health. The authors thank Dr Mark Raffeld and Dr David E. Kleiner from the National Cancer Institute for their help with immunostaining, and the NIH Intramural Sequencing Center (NISC) for performing next-generation sequencing.

\section{Author Contributions}

All authors made a significant contribution to the work reported, whether that is in the conception, study design, execution, acquisition of data, analysis and interpretation, or in all these areas; took part in drafting, revising or critically reviewing the article; gave final approval of the version to be published; have agreed on the journal to which the article has been submitted; and agree to be accountable for all aspects of the work.

\section{Funding}

This research received no external funding.

\section{Disclosure}

No potential conflicts of interest were disclosed by the authors.

\section{References}

1. Kulik L, El-Serag HB. Epidemiology and management of hepatocellular carcinoma. Gastroenterology. 2019;156(2):477-491 e471. doi:10.1053/j.gastro.2018.08.065

2. McGlynn KA, Petrick JL, El-Serag HB. Epidemiology of hepatocellular carcinoma. Hepatology. 2021;73(Suppl 1):4-13. doi:10.1002/ hep. 31288

3. Ryerson AB, Eheman CR, Altekruse SF, et al. Annual Report to the Nation on the Status of Cancer, 1975-2012, featuring the increasing incidence of liver cancer. Cancer. 2016;122(9):1312-1337. doi:10.1002/cncr.29936

4. Ghouri YA, Mian I, Rowe JH. Review of Hepatocellular carcinoma: epidemiology, etiology, and carcinogenesis. J Carcinog. 2017;16:1. doi:10.4103/jcar.JCar_9_16

5. Disease GBD, Injury I, Prevalence C. Global, regional, and national incidence, prevalence, and years lived with disability for 354 diseases and injuries for 195 countries and territories, 1990-2017: a systematic analysis for the Global Burden of Disease Study 2017. Lancet. 2018;392(10159):1789-1858.

6. Bouvard V, Baan R, Straif K, et al. A review of human carcinogensPart B: biological agents. Lancet Oncol. 2009;10(4):321-322. doi:10.1016/S1470-2045(09)70096-8

7. El-Serag HB, Davila JA. Surveillance for hepatocellular carcinoma: in whom and how? Therap Adv Gastroenterol. 2011;4(1):5-10. doi: $10.1177 / 1756283 \times 10385964$

8. Ogata F, Kobayashi M, Akuta N, et al. Outcome of All-Oral Direct-Acting Antiviral Regimens on the Rate of Development of Hepatocellular Carcinoma in Patients with Hepatitis C Virus Genotype 1-Related Chronic Liver Disease. Oncology. 2017;93 (2):92-98. doi:10.1159/000470910

9. Lindenbach BD, Rice CM. Unravelling hepatitis C virus replication from genome to function. Nature. 2005;436(7053):933-938. doi:10.1038/nature04077

10. Lemon SM, McGivern DR. Is hepatitis C virus carcinogenic? Gastroenterology. 2012;142(6):1274-1278. doi:10.1053/j. gastro.2012.01.045

11. Shlomai A, de Jong YP, Rice CM. Virus associated malignancies: the role of viral hepatitis in hepatocellular carcinoma. Semin Cancer Biol. 2014;26:78-88. doi:10.1016/j.semcancer. 2014.01.004

12. Moriya K, Fujie H, Shintani Y, et al. The core protein of hepatitis $\mathrm{C}$ virus induces hepatocellular carcinoma in transgenic mice. Nat Med. 1998;4(9):1065-1067. doi:10.1038/2053

13. Szmuness W, Stevens CE, Ikram H, Much MI, Harley EJ, Hollinger B. Prevalence of hepatitis B virus infection and hepatocellular carcinoma in Chinese-Americans. J Infect Dis. 1978;137 (6):822-829. doi:10.1093/infdis/137.6.822

14. Geier A, Gartung C, Dietrich CG. Hepatitis B e Antigen and the Risk of Hepatocellular Carcinoma. $N$ Engl $J$ Med. 2002;347 (21):1721-1722.

15. Brechot C, Gozuacik D, Murakami Y, Paterlini-Brechot P. Molecular bases for the development of hepatitis B virus (HBV)-related hepatocellular carcinoma (HCC). Semin Cancer Biol. 2000;10 (3):211-231. doi:10.1006/scbi.2000.0321

16. Tu T, Budzinska MA, Shackel NA, Urban S. HBV DNA Integration: molecular Mechanisms and Clinical Implications. Viruses. 2017;9 (4):75. doi:10.3390/v9040075

17. Llovet JM, Zucman-Rossi J, Pikarsky E, et al. Hepatocellular carcinoma. Nat Rev Dis Primers. 2016;2:16018.

18. Cancer Genome Atlas Research Network. Electronic address wbe, Cancer Genome Atlas Research N. Comprehensive and Integrative Genomic Characterization of Hepatocellular Carcinoma. Cell. 2017;169(7):1327-1341 e1323. doi:10.1016/j. cell.2017.05.046 
19. Hoshida Y, Nijman SM, Kobayashi M, et al. Integrative transcriptome analysis reveals common molecular subclasses of human hepatocellular carcinoma. Cancer Res. 2009;69(18):7385-7392. doi:10.1158/0008-5472.CAN-09-1089

20. Kurebayashi Y, Ojima H, Tsujikawa H, et al. Landscape of immune microenvironment in hepatocellular carcinoma and its additional impact on histological and molecular classification. Hepatology. 2018;68(3):1025-1041. doi:10.1002/hep.29904

21. Sia D, Jiao Y, Martinez-Quetglas I, et al. Identification of an Immune-specific Class of Hepatocellular Carcinoma, Based on Molecular Features. Gastroenterology. 2017;153(3):812-826. doi:10.1053/j.gastro.2017.06.007

22. Pinato DJ, Guerra N, Fessas $P$, et al. Immune-based therapies for hepatocellular carcinoma. Oncogene. 2020;39(18):3620-3637. doi:10.1038/s41388-020-1249-9

23. Johnston MP, Khakoo SI. Immunotherapy for hepatocellular carcinoma: current and future. World J Gastroenterol. 2019;25 (24):2977-2989. doi:10.3748/wjg.v25.i24.2977

24. Zhou G, Sprengers D, Boor PPC, et al. Antibodies Against Immune Checkpoint Molecules Restore Functions of Tumor-Infiltrating T Cells in Hepatocellular Carcinomas. Gastroenterology. 2017;153 (4):1107-1119 e1110. doi:10.1053/j.gastro.2017.06.017

25. Sekhar V, Pollicino T, Diaz G, et al. Infection with hepatitis $\mathrm{C}$ virus depends on TACSTD2, a regulator of claudin-1 and occludin highly downregulated in hepatocellular carcinoma. PLoS Pathog. 2018;14 (3):e1006916. doi:10.1371/journal.ppat.1006916

26. Melis M, Diaz G, Kleiner DE, et al. Viral expression and molecular profiling in liver tissue versus microdissected hepatocytes in hepatitis B virus-associated hepatocellular carcinoma. $J$ Transl Med. 2014;12:230. doi:10.1186/s12967-014-0230-1

27. Farci P, Diaz G, Chen Z, et al. B cell gene signature with massive intrahepatic production of antibodies to hepatitis B core antigen in hepatitis B virus-associated acute liver failure. Proc Natl Acad Sci $U S$ A. 2010;107(19):8766-8771. doi:10.1073/pnas.1003854107

28. Kechin A, Boyarskikh U, Kel A, Filipenko M. cutPrimers: a New Tool for Accurate Cutting of Primers from Reads of Targeted Next Generation Sequencing. J Comput Biol. 2017;24(11):1138-1143. doi:10.1089/cmb.2017.0096

29. Dobin A, Davis CA, Schlesinger F, et al. STAR: ultrafast universal RNA-seq aligner. Bioinformatics. 2013;29(1):15-21. doi:10.1093/ bioinformatics/bts635
30. Li B, Dewey CN. RSEM: accurate transcript quantification from RNA-Seq data with or without a reference genome. $B M C$ Bioinform. 2011;12:323. doi:10.1186/1471-2105-12-323

31. Ritchie ME, Phipson B, Wu D, et al. limma powers differential expression analyses for RNA-sequencing and microarray studies. Nucleic Acids Res. 2015;43(7):e47. doi:10.1093/nar/gkv007

32. Fabregat A, Jupe S, Matthews L, et al. The Reactome Pathway Knowledgebase. Nucleic Acids Res. 2018;46(D1):D649-D655. doi:10.1093/nar/gkx1132

33. Wang J, Vasaikar S, Shi Z, Greer M, Zhang B. WebGestalt 2017: a more comprehensive, powerful, flexible and interactive gene set enrichment analysis toolkit. Nucleic Acids Res. 2017;45(W1):W130W137. doi:10.1093/nar/gkx356

34. Harouaka D, Engle RE, Wollenberg K, et al. Diminished viral replication and compartmentalization of hepatitis $\mathrm{C}$ virus in hepatocellular carcinoma tissue. Proc Natl Acad Sci U S A. 2016;113(5):1375-1380. doi:10.1073/pnas.1516879113

35. Diaz G, Engle RE, Tice A, et al. Molecular signature and mechanisms of hepatitis D virus-associated hepatocellular carcinoma. Mol Cancer Res. 2018;16(9):1406-1419. doi:10.1158/1541-7786.MCR18-0012

36. Hirayasu K, Arase H. Functional and genetic diversity of leukocyte immunoglobulin-like receptor and implication for disease associations. J Hum Genet. 2015;60(11):703-708. doi:10.1038/ jhg.2015.64

37. Mantovani A, Marchesi F, Malesci A, Laghi L, Allavena P. Tumourassociated macrophages as treatment targets in oncology. Nat Rev Clin Oncol. 2017;14(7):399-416. doi:10.1038/nrclinonc.2016.217

38. Rehermann B, Nascimbeni M. Immunology of hepatitis B virus and hepatitis C virus infection. Nat Rev Immunol. 2005;5(3):215-229. doi:10.1038/nri1573

39. Kroy DC, Ciuffreda D, Cooperrider JH, et al. Liver environment and HCV replication affect human T-cell phenotype and expression of inhibitory receptors. Gastroenterology. 2014;146(2):550-561. doi:10.1053/j.gastro.2013.10.022

40. Ackermann C, Smits M, Woost R, et al. HCV-specific CD4+ T cells of patients with acute and chronic $\mathrm{HCV}$ infection display high expression of TIGIT and other co-inhibitory molecules. Sci Rep. 2019;9(1):10624. doi:10.1038/s41598-019-47024-8
Journal of Hepatocellular Carcinoma

\section{Publish your work in this journal}

The Journal of Hepatocellular Carcinoma is an international, peerreviewed, open access journal that offers a platform for the dissemination and study of clinical, translational and basic research findings in this rapidly developing field. Development in areas including, but not limited to, epidemiology, vaccination, hepatitis therapy, pathology and molecular tumor classification and prognostication are all considered for publication. The manuscript management system is completely online and includes a very quick and fair peer-review system, which is all easy to use. Visit http://www.dovepress.com/ testimonials.php to read real quotes from published authors. 